\title{
Effect of acceleration forces during transport through a pneumatic tube system on ROTEM $^{\circledR}$ analysis
}

\author{
Gerhard Amann ${ }^{1}$, Christine Zehntner ${ }^{1}$, Florian Marti ${ }^{1}$ \\ and Giuseppe Colucci ${ }^{2}$,* \\ ${ }^{1}$ Department of Anaesthesiology and Pain Therapy, \\ Kantonsspital Winterthur, Winterthur, Switzerland \\ ${ }^{2}$ Department of Haematology and Central Haematology \\ Laboratory, Inselspital, Bern University Hospital and \\ University of Bern, Switzerland
}

\begin{abstract}
Background: ROTEM ${ }^{\circledR}$ is considered a helpful point-of-care device to monitor blood coagulation in emergency situations. Centrally performed analysis is desirable but rapid transport of blood samples is an important prerequisite. The effect of acceleration forces on sample transport through a pneumatic tube system on ROTEM $^{\circledR}$ should be tested at each institution to exclude a pre-analytical influence. The aims of the present work were: (i) to investigate the effect of pneumatic tube transport on ROTEM $^{\circledR}$ parameters; (ii) to compare blood sample transport via pneumatic tube vs. manual transportation; and (iii) to determine the effect of acceleration forces on ROTEM $^{\circledR}$ parameters.
\end{abstract}

Methods: This is a single centre study with 20 healthy volunteers. Five whole blood samples were transferred to the central haematology laboratory by either normal transport or pneumatic delivery with different speed and acceleration forces. EXTEM, INTEM, FIBTEM and APTEM were analysed in parallel with two ROTEM ${ }^{\circledR}$ devices and compared. Acceleration forces were measured during transport with two different instruments.

Results: Increment of transport time, speed and distance resulted in an augmentation of acceleration forces and peaks. All results of the ROTEM ${ }^{\circledR}$ analysis after manual transport or pneumatic delivery were within normal range. However, increase in acceleration forces resulted in minimally but statistically significant changes in multiple ROTEM $^{\circledR}$ parameters. The higher the acceleration forces, the more ROTEM ${ }^{\circledR}$ parameters are influenced.

Conclusions: Acceleration forces during transport through a pneumatic tube system have an influence on ROTEM $^{\circledR}$ parameters. Prior to transfer blood samples via pneumatic tube system these influences should be tested to exclude clinically relevant blood coagulation activation in vitro.

*Corresponding author: Giuseppe Colucci, Department of Haematology and Central Haematology Laboratory Inselspital, Bern University Hospital and University of Bern, Freiburgstrasse 10, 3010 Bern, Switzerland

Phone: +41 31 6320264, Fax: +41316323406,

E-mail: giuseppe.colucci@insel.ch

Received October 31, 2011; accepted February 6, 2012;

previously published online March 9, 2012
Keywords: acceleration forces; coagulation; laboratory based ROTEM $^{\circledR}$; pneumatic tube transport; point-of-care.

\section{Introduction}

The modified rotation thrombelastometry $\left(\right.$ ROTEM $\left.^{\circledR}\right)$ was developed as a point-of-care coagulation monitoring device. The automatised system allows determination of the coagulation proprieties of whole blood during clot formation and lysis (1). With simple handling the method enables rapid analysis and results are graphically visualised live on a display. The device shows four pictures named EXTEM, INTEM, FIBTEM and APTEM which depict the clotting process after activation with different agonist. The coagulation is activated either with ellagic acid, which leads to a standardised activation of the contact phase of the intrinsic system (INTEM) or thromboplastin from rabbit brain which is used for the activation of the extrinsic coagulation system (EXTEM). In addition, reagents, such as aprotinin (APTEM) or cytochalasin D (FIBTEM) are used in order to study the EXTEM with (in vitro) inhibition of fibrinolytic activity (APTEM) and the EXTEM with inhibition of platelets (FIBTEM), respectively. Results are available 10-15 min after blood collection. The device is fast and is helpful especially in emergency situations with life-threatening bleeding (2). The four pictures should enable the coagulation defect to be identified and a therapeutic option to be found. During emergency treatment, the simultaneous execution of ROTEM ${ }^{\circledR}$ analysis represents an additional task for the attending team. The ROTEM ${ }^{\circledR}$ delta device allows real time transmission of on-going ROTEM $^{\circledR}$ results to different sites without time delay. This option encourages installing and performing ROTEM $^{\circledR}$ analysis in a central laboratory. Consequently, rapid transport of blood samples to a central laboratory is a prerequisite. Automated transport systems have been developed to reduce the turnaround time and workload. Pneumatic tube systems are widely used in hospitals (3). Transport of blood samples through the pneumatic tube system may be advantageous also for ROTEM ${ }^{\circledR}$ analysis. However, samples in the pneumatic tube are subjected to acceleration and deceleration forces necessary to achieve high speed in order to cover long distances quickly. Pneumatic tube systems vary in diameter, size of the capsule, number of lines and transfer stations as well as forces to achieve the necessary speed. These forces on blood samples are known to cause certain cell damage influencing routine haematology results $(4,5)$. Significant changes in lactate dehydrogenase concentrations (6), effects on blood gas measurements (7-9) and on thromboelastographic analysis with the TEG $5000^{\circledR}$ (4) have been described. Pre-analytical effects of pneumatic tube transport on coagulation parameters and 
platelet function have also been evaluated (10-12). Finally, each pneumatic transport system possesses a unique technical and structural pattern and should be tested for pre-analytical effects (13), including effects with regard to ROTEM ${ }^{\circledast}$ analysis (14). Indeed the manufacturer of the ROTEM ${ }^{\circledast}$ delta device advises against the transport of blood samples via pneumatic tube unless the influence of local mechanical transport system is investigated.

The aims of the present work were: (i) to investigate the effect of pneumatic tube transport on ROTEM $^{\circledast}$ parameters; (ii) to compare blood sample transport via pneumatic tube vs. manual transport; and (iii) to determine the effect of acceleration forces on ROTEM ${ }^{\circledast}$ parameters.

\section{Materials and methods}

The study was performed at the Kantonsspital Winterthur after approval from the Local Ethics Committee (Kantonale Ethikkommission Zürich, KEK-ZH-Nr. 2011-0222). After informed consent, 20 healthy volunteers were recruited and prospectively enrolled by medical personal at our institution. Exclusion criteria were: (i) age under 18 years; (ii) systolic blood pressure below $100 \mathrm{~mm} \mathrm{Hg}$; (iii) known coagulation disorders; (iv) illness or surgery in the last 4 weeks; (v) transfusion in the last 12 months; and (vi) intake of any medication including contraceptives and analgesics. Whole blood samples were collected from an anticubital vein into five $4.3 \mathrm{~mL}$ plastic vials (S-Monovette ${ }^{\circledR} 4.3 \mathrm{~mL}$ 0NC; Sarstedt, Nümbrecht, Germany) containing $0.43 \mathrm{~mL} 0.106 \mathrm{~mol} / \mathrm{L}$ trisodium citrate solution. All samples were collected in the central operating unit and labelled A, B, C, D and E, respectively. Sample A was delivered manually to the central haematology laboratory at room temperature. Sample B was sent by pneumatic tube system with a velocity of $3 \mathrm{~m} / \mathrm{s}$ according to local standard "priority" mode. Samples C, D and E were sent through the pneumatic tube system with a velocity of $7 \mathrm{~m} / \mathrm{s}$ for $270 \mathrm{~m}, 540 \mathrm{~m}$ and $1080 \mathrm{~m}$, respectively (Table 1).

\section{Pneumatic tube system and acceleration forces}

The pneumatic tube system at the Kantonsspital Winterthur (Transmatic MC 14/NW 125; Transro AG, Wohlen, Switzerland) is a one-tube-system consisting of 14 lines with intersection at two transfer stations. Blood samples sent to the central laboratory have a standard "priority" mode (direct transfer) and a velocity of $3 \mathrm{~m} / \mathrm{s}$. The capsules sent to destinations outside the central laboratory have a velocity of $7 \mathrm{~m} / \mathrm{s}$. To measure the acceleration forces during pneumatic delivery, we used two devices: IPod touch (Apple ${ }^{\circledR}$ Inc.; Cupertino, USA) with an associated application (SensorData, Wavefront Labs ${ }^{\odot}$; distributed by Apple ${ }^{\circledR}$ Inc.; Cupertino, USA) which measures linear and rotation acceleration forces with a frequency of $100 \mathrm{~Hz}$ (see Supplemental data Figure 1A; accompanies the online version of this article at http://www.degruyter.com/view/j/cclm.2012.50. issue-8/issue-files/cclm.2012.50.issue-8.xml). This device was fixed to the capsule (see Supplemental data Figure 2A). Because the linear accelerations measured using the IPod are limited to maximal $2 \mathrm{~g}$ (unit of measure of acceleration due to gravity at the earth's surface) we used a second device. The second device was a self recording impact accelerometer X250-2 (Gulf Coast Data Concepts ${ }^{\odot ;}$ Waveland, USA) which measures with a frequency of $256 \mathrm{~Hz}$. The latter is very light (33 g, see Supplemental data Figure 1B) and was placed inside the capsule close to the blood sample (see Supplemental data Figure 2B). X250-2 measures forces in the range of $\pm 28 \mathrm{~g}$. The two devices measured accelerations in three axes $\mathrm{x}, \mathrm{y}, \mathrm{z}$, and the raw data have to be corrected for an offset value (for details see Supplemental data). Acceleration forces are expressed as area under the curve (AUC) and numbers of acceleration peaks (n).

\section{ROTEM $^{\circledR}$ analysis}

Analysis of all samples was performed using two identical ROTEM ${ }^{\circledR}$ delta devices $\left(\right.$ ROTEM $^{\circledR} 1$ and ROTEM ${ }^{\circledR}$ 2; Axon Lab AG, Baden, Switzerland) placed at the central haematology laboratory of the Kantonsspital Winterthur. Quality control of the devices was performed initially following the internal quality protocol with the respective reagent (ROTROL N; Pentapharm AG Basel, Switzerland). All tests were performed by the first author of this study, who was initially trained and supervised by qualified laboratory staff. Prior to ROTEM ${ }^{\circledR}$ analysis, all samples were placed over the ROTEM $^{\circledR}$ delta devices at $37^{\circ} \mathrm{C}$ for $5 \mathrm{~min}$ and all measurements were performed at this temperature. The reagents, stored at temperatures between $4^{\circ} \mathrm{C}$ and $8^{\circ} \mathrm{C}$, were kept at room temperature for $10 \mathrm{~min}$ and shaken gently before pipetted. INTEM, EXTEM, FIBTEM and APTEM test were performed as described previously (14). For each analysis standard ROTEM ${ }^{\circledR}$ parameters, such as clotting time (CT, s), A5 (clot thickness after 5 min, $\mathrm{mm}$ ), A10 (clot thickness after $10 \mathrm{~min}, \mathrm{~mm}$ ), A15 (clot thickness after $15 \mathrm{~min}, \mathrm{~mm}$ ), A30 (clot thickness after $30 \mathrm{~min}, \mathrm{~mm}$ ), clot formation time (CFT, s), maximum clot firmness (MCF, mm), alpha ( $\alpha$, angle) LI30 (lysis index after $30 \mathrm{~min}$ ), LI45 (lysis index after $45 \mathrm{~min}$ ), ML (maximal lysis) and AUC (area under the curve) were measured. These parameters describe: (i) the period from analysis start until a $2 \mathrm{~mm}$ amplitude occurs (CT); (ii) the subsequent period until an amplitude of $20 \mathrm{~mm}$ is reached (CFT); (iii) the kinetics of clot formation ( $\alpha$ ); (iv) the clot firmness at different time points (A5 after $5 \mathrm{~min}, \mathrm{~A} 10$ after 10 min, A15 after $15 \mathrm{~min}$ and A30 after $30 \mathrm{~min}$ ); (v) the maximum firmness that the clot achieves during the analysis (MCF); (vi) the fibrinolysis at particular time point (LI30 after $30 \mathrm{~min}$, LI45 after $45 \mathrm{~min}$ ); and (vii) the maximum fibrinolysis detected (ML).

\section{Statistical analysis}

All quantitative data are expressed as median and interquartile range (IQR). Non-parametric statistic was applied and performed with SPSS Statistics Version $19^{\circledR}$ software (SPSS Inc., Chicago,

Table 1 Study design (results expressed as median and interquartile range, IQR).

\begin{tabular}{llcclcc}
\hline & Delivery mode & Total distance & Speed & $\begin{array}{l}\text { Pneumatic } \\
\text { transport time }\end{array}$ & $\begin{array}{l}\text { Linear acceleration } \\
\text { forces (AUC) }\end{array}$ & $\begin{array}{l}\text { Linear acceleration } \\
\text { peaks }>25 g(\mathrm{n})\end{array}$ \\
\hline Sample A & Manual & $50 \mathrm{~m}$ & Walk & - & - & - \\
Sample B & Pneumatic priority & $270 \mathrm{~m}$ & $3 \mathrm{~m} / \mathrm{s}$ & $135 \mathrm{~s}(117-149)$ & $95.9(93.3-99.3)$ & $3.5(3-4)$ \\
Sample C & Pneumatic normal & $270 \mathrm{~m}$ & $7 \mathrm{~m} / \mathrm{s}$ & $176 \mathrm{~s}(152-203)$ & $116.3(114.5-117.7)$ & $4.5(4-8)$ \\
Sample D & Pneumatic normal & $540 \mathrm{~m}$ & $7 \mathrm{~m} / \mathrm{s}$ & $334 \mathrm{~s}(315-390)$ & $213.1(208.2-223.3)$ & $9.0(5-10)$ \\
Sample E & Pneumatic normal & $1080 \mathrm{~m}$ & $7 \mathrm{~m} / \mathrm{s}$ & $652 \mathrm{~s}(568-852)$ & $413.2(406.5-427.3)$ & $18.5(13-22)$ \\
\hline
\end{tabular}


IL, USA), SigmaPlot 11.0 (Systat Software, Inc., Chicago, USA) and Microsoft Excel $2010^{\odot}$ (Microsoft Corporation; Redmont, OR, USA). Comparisons were performed with the Wilcoxon signed rank test. A two-tailed $\mathrm{p}$ value of $<0.05$ was considered significant. The offset correction of the X250-2 logger, the AUC of the acceleration vectors and the peaks were calculated with Octave 3.2.4 (Free Software Foundation; Boston, MA, USA).

\section{Results}

Twenty healthy volunteers [median age 41.4 years (range 27-52), comprising 8 women [median age 47 years (range 2852)] and 12 men [median age 34 years (range 27-49)]] were enrolled. All citrated blood samples were labelled A, B, C, D, $\mathrm{E}$, respectively and sent through pneumatic delivery system with a defined velocity (samples B-E) or hand carried to central laboratory (sample A, Table 1). Median transport time of sample A was 157 seconds (s) (IQR: 147-169 s). Median transport time in the pneumatic tube was $135 \mathrm{~s}$ (IQR: 117-149 s) for sample B, 176 s (IQR: 152-203 s) for sample C, 334 s (IQR: 315-390 s) for sample D, $652 \mathrm{~s}$ (IQR: 568-852 s) for sample $\mathrm{E}$, respectively. The rotation acceleration force measured with IPod (median AUC, rad/s) was 3745 (IQR: 3674-3833, range $4.24 \%$ ) for sample B, 4400 (IQR: 4342-4460, range 2.67\%) for sample C, 8557 (IQR: 8526-8602, range 0.88\%) for sample D and 17249 (IQR: 17101-17353, range 1.47\%) for sample E, respectively. The linear acceleration forces (median AUC, $\mathrm{m} / \mathrm{s}$ ) measured with the device placed inside the capsule (X250-2) was 95.9 (IQR: 93.9-99.3, range 5.59\%) for sample B, 116.3 (IQR: 114.5-117.7, range 2.74\%) for sample C, 213.1 (IQR: 208.1-223.3, range 7.13\%) for sample D and 413.2 (IQR: 406.5-427.3, range 5.03\%) for sample E, respectively. Linear acceleration peaks are summarised in Tables 1 and 2. Median time between blood collection and start of ROTEM ${ }^{\circledR}$ test was $11 \mathrm{~min}$ (IQR: 10-12 min) for sample A, $17 \mathrm{~min}$ (IQR: 16-20 $\mathrm{min}$ ) for sample B, $81 \mathrm{~min}$ (IQR: 79-86 min) for sample C, 130 min (IQR: 126-144 min) for sample D and $157 \mathrm{~min}$ (IQR: 151-160 min) for sample E, respectively.

Table 2 Linear acceleration peaks measured during tube transport delivery (n, median and IQR).

\begin{tabular}{llllll}
\hline & Sample A & Sample B & Sample C & Sample D & Sample E \\
\hline$>1 g$ & - & $7928(7634-8119)$ & $9791(9319-10247)$ & $18143(17817-19822)$ & $35676(35138-37934)$ \\
$>2 g$ & - & $2445(2331-2551)$ & $3399(3048-3581)$ & $6118(5689-6671)$ & $11551(11432-12462)$ \\
$>4 g$ & - & $598(545-627)$ & $835(767-903)$ & $1526(1476-1599)$ & $3027(2833-3208)$ \\
$>8 g$ & - & $118(95-132)$ & $186(158-216)$ & $331(232-431)$ & $748(545-806)$ \\
$>16 g$ & - & $19.0(16-23)$ & $31.5(22-45)$ & $51(29-76)$ & $126(89-142)$ \\
$>25 g$ & - & $3.5(3-4)$ & $4.5(4-8)$ & $9.0(5-10)$ & $18.5(13-22)$ \\
\hline
\end{tabular}

Table 3 Results of thromboelastometry: manual transport (sample A) and pneumatic delivery (3 m/s, $270 \mathrm{~m}$ : sample B): median and interquartile range (IQR).

\begin{tabular}{|c|c|c|c|c|c|c|}
\hline & \multicolumn{3}{|l|}{ EXTEM } & \multicolumn{3}{|l|}{ INTEM } \\
\hline & Sample A (manual) & Sample B & p-Value & Sample A (manual) & Sample B & $\mathrm{p}$-Value \\
\hline $\mathrm{CT}$ & $50(47-54)$ & $51(46-53)$ & 0.738 & $176(165-202)$ & $169(158-185)$ & 0.245 \\
\hline A5 & $45(41-48)$ & $46(42-48)$ & 0.263 & $46(43-49)$ & $46(43-49)$ & 0.258 \\
\hline A10 & $56(52-58)$ & $56(52-57)$ & 0.021 & $55(53-58)$ & $55(52-57)$ & 0.182 \\
\hline A15 & $60(57-62)$ & $59(56-60)$ & $<0.001$ & $59(56-62)$ & $59(56-60)$ & 0.024 \\
\hline A30 & $62(59-64)$ & $61(58-63)$ & $<0.001$ & $60(57-62)$ & $58(56-61)$ & 0.004 \\
\hline CFT & $86(76-102)$ & 87 (77-97) & 0.734 & $66(58-81)$ & $66(58-77)$ & 0.869 \\
\hline MCF & $62(60-64)$ & $61(59-63)$ & $<0.001$ & $62(57-62)$ & $60(57-61)$ & 0.027 \\
\hline$\alpha$ & $73(70-74)$ & $72(71-75)$ & 0.839 & $77(74-78)$ & $77(75-78)$ & 0.953 \\
\hline \multirow[t]{3}{*}{ AUC } & $6212(5933-6382)$ & $6089(5858-6249)$ & $<0.001$ & $6118(5678-6212)$ & $5964(5640-6087)$ & 0.033 \\
\hline & \multicolumn{3}{|l|}{ FIBTEM } & \multicolumn{3}{|l|}{ APTEM } \\
\hline & $\underline{\text { Sample A (manual) }}$ & Sample B & $\mathrm{p}$-Value & Sample A (manual) & Sample B & $\mathrm{p}$-Value \\
\hline CT & $46(44-48)$ & $47(43-49)$ & 0.922 & $51(48-54)$ & $48(47-55)$ & 0.756 \\
\hline A5 & $10(10-13)$ & $12(10-13)$ & 0.241 & $46(40-49)$ & $44(43-48)$ & 0.241 \\
\hline A10 & $11(11-13)$ & $12(10-13)$ & 0.277 & $55(51-58)$ & $55(51-57)$ & 0.135 \\
\hline A15 & $12(11-14)$ & $13(10-13)$ & 0.715 & $60(57-62)$ & $59(55-61)$ & 0.068 \\
\hline A30 & $12(11-13)$ & $13(11-14)$ & 0.561 & $62(58-64)$ & $61(57-62)$ & $<0.001$ \\
\hline CFT & - & - & - & $88(73-105)$ & 89 (76-97) & 0.277 \\
\hline MCF & $12(11-13)$ & $13(11-14)$ & 0.706 & $62(58-64)$ & $61(58-63)$ & 0.021 \\
\hline$\alpha$ & $68(63-72)$ & $67(64-70)$ & 0.829 & $72(69-75)$ & $72(71-74)$ & 0.326 \\
\hline AUC & $1183(1112-1350)$ & $1222(1078-1335)$ & 0.674 & $6209(5818-6355)$ & $6044(5775-6248)$ & 0.008 \\
\hline
\end{tabular}

ROTEM $^{\circledR}$ parameters: clotting time (CT, s), clot thickness (A5 after 5 min, A10 after 10 min, A15 after 15 min, A30 after 30 min, respectively, $\mathrm{mm}$ ), clot formation time (CFT, s), maximum clot firmness (MCF, mm), alpha ( $\alpha$, angle) and AUC (area under the curve). 
Table 4 Results of thromboelastometry: manual transport (sample A) and pneumatic delivery (7 m/s, $270 \mathrm{~m}$ : sample C): median and interquartile range (IQR).

\begin{tabular}{|c|c|c|c|c|c|c|}
\hline & \multicolumn{3}{|l|}{ EXTEM } & \multicolumn{3}{|l|}{ INTEM } \\
\hline & Sample A (manual) & Sample C & p-Value & Sample A (manual) & Sample C & $\mathrm{p}$-Value \\
\hline CT & $50(47-54)$ & $51(48-53)$ & 0.784 & $176(165-202)$ & $160(152-182)$ & 0.165 \\
\hline A5 & $45(41-48)$ & $44(41-47)$ & 0.001 & $46(43-49)$ & $45(43-48)$ & 0.089 \\
\hline A10 & $56(52-58)$ & $54(51-57)$ & $<0.001$ & $55(53-58)$ & $54(52-57)$ & 0.015 \\
\hline A15 & $60(57-62)$ & $57(55-60)$ & $<0.001$ & $59(56-62)$ & $57(56-60)$ & $<0.001$ \\
\hline A30 & $62(59-64)$ & $60(57-62)$ & $<0.001$ & $60(57-62)$ & $58(56-60)$ & $<0.001$ \\
\hline CFT & $86(76-102)$ & $88(73-98)$ & 0.347 & $66(58-81)$ & $66(58-74)$ & 0.784 \\
\hline $\mathrm{MCF}$ & $62(60-64)$ & $61(58-62)$ & $<0.001$ & $62(57-62)$ & $60(57-61)$ & $<0.001$ \\
\hline$\alpha$ & $73(70-74)$ & $72(70-75)$ & 0.893 & $77(74-78)$ & $77(75-78)$ & 0.632 \\
\hline \multirow[t]{3}{*}{ AUC } & $6212(5933-6382)$ & $6024(5743-6173)$ & $<0.001$ & 6118 (5678-6212) & $5890(5638-6050)$ & $<0.001$ \\
\hline & \multicolumn{3}{|l|}{ FIBTEM } & \multicolumn{3}{|l|}{ APTEM } \\
\hline & Sample A (manual) & Sample C & $\mathrm{p}$-Value & Sample A (manual) & Sample C & $\overline{\mathrm{p} \text {-Value }}$ \\
\hline $\mathrm{CT}$ & $46(44-48)$ & $47(44-48)$ & 0.671 & $51(48-54)$ & $52(48-55)$ & 0.812 \\
\hline A5 & $10(10-13)$ & $11(10-12)$ & 1.000 & $46(40-49)$ & $43(40-47)$ & 0.004 \\
\hline A10 & $11(11-13)$ & $12(10-13)$ & 0.839 & $55(51-58)$ & $53(50-56)$ & $<0.001$ \\
\hline A15 & $12(11-14)$ & $12(11-13)$ & 0.520 & $60(57-62)$ & $57(54-60)$ & $<0.001$ \\
\hline A30 & $12(11-13)$ & $12(11-13)$ & 0.569 & $62(58-64)$ & $60(56-62)$ & $<0.001$ \\
\hline CFT & - & - & - & $88(73-105)$ & $88(73-99)$ & 0.003 \\
\hline $\mathrm{MCF}$ & $12(11-13)$ & $12(11-13)$ & 0.110 & $62(58-64)$ & $60(57-62)$ & $<0.001$ \\
\hline$\alpha$ & $68(63-72)$ & $69(65-72)$ & 0.252 & $72(69-75)$ & $72(70-75)$ & 0.013 \\
\hline AUC & $1183(1112-1350)$ & $1158(1081-1341)$ & 0.349 & $6209(5818-6355)$ & $5984(5679-6175)$ & $<0.001$ \\
\hline
\end{tabular}

ROTEM $^{\circledR}$ parameters: clotting time (CT, s), clot thickness (A5 after 5 min, A10 after 10 min, A15 after 15 min, A30 after 30 min, respectively, $\mathrm{mm}$ ), clot formation time (CFT, s), maximum clot firmness (MCF, mm), alpha ( $\alpha$, angle) and AUC (area under the curve).

Table 5 Results of thromboelastometry: manual transport (sample A) and pneumatic delivery (7 m/s, 1080 m: sample E): median and interquartile range (IQR).

\begin{tabular}{|c|c|c|c|c|c|c|}
\hline & \multicolumn{3}{|l|}{ EXTEM } & \multicolumn{3}{|l|}{ INTEM } \\
\hline & Sample A (manual) & Sample E & p-Value & Sample A (manual) & Sample E & p-Value \\
\hline $\mathrm{CT}$ & $50(47-54)$ & $48(44-49)$ & 0.008 & $176(165.25-201.5)$ & $156(150-177)$ & 0.058 \\
\hline A5 & $45(41-48)$ & $43(40-46)$ & $<0.001$ & $46(43-49)$ & $45(41-48)$ & 0.003 \\
\hline A10 & $56(52-58)$ & $53(50-55)$ & $<0.001$ & $55(53-58)$ & $54(51-56)$ & $<0.001$ \\
\hline A15 & $60(57-62)$ & $57(55-60)$ & $<0.001$ & $59(56-62)$ & $57(54-59)$ & $<0.001$ \\
\hline $\mathrm{A} 30$ & $62(59-64)$ & $60(56-61)$ & $<0.001$ & $60(57-62)$ & $58(53-60)$ & $<0.001$ \\
\hline CFT & $86(76-102)$ & $91(80-103)$ & 0.442 & $66(58-81)$ & $72(60-84)$ & 0.202 \\
\hline $\mathrm{MCF}$ & $62(59-64)$ & $60(57-62)$ & $<0.001$ & $62(57-62)$ & $59(54-60)$ & $<0.001$ \\
\hline$\alpha$ & $73(70-75)$ & $72(69-74)$ & 0.301 & $76(74-78)$ & $76(74-78)$ & 0.890 \\
\hline \multirow[t]{3}{*}{ AUC } & $6212(5933-6382)$ & $5983(5636-6159)$ & $<0.001$ & $6118(5678-6212)$ & $5872(5410-6015)$ & $<0.001$ \\
\hline & \multicolumn{3}{|l|}{ FIBTEM } & \multicolumn{3}{|l|}{ APTEM } \\
\hline & Sample A (manual) & Sample E & p-Value & Sample A (manual) & Sample E & $\mathrm{p}$-Value \\
\hline $\mathrm{CT}$ & $46(44-48)$ & $44(41-47)$ & 0.001 & $51(48-54)$ & $49(46-51)$ & 0.096 \\
\hline A5 & $10(10-13)$ & $11(10-12)$ & 0.542 & $46(40-49)$ & $43(39-46)$ & $<0.001$ \\
\hline A10 & $11(11-13)$ & $11(11-12)$ & 0.080 & $55(51-58)$ & $52(50-55)$ & $<0.001$ \\
\hline A15 & $12(11-14)$ & $12(11-13)$ & 0.173 & $60(57-62)$ & $56(54-59)$ & $<0.001$ \\
\hline $\mathrm{A} 30$ & $12(11-14)$ & $13(11-14)$ & 0.678 & $62(58-64)$ & $59(56-61)$ & $<0.001$ \\
\hline CFT & - & - & - & $88(73-105)$ & $91(76-104)$ & 0.490 \\
\hline $\mathrm{MCF}$ & $12(11-13)$ & $12(11-13)$ & 0.263 & $62(58-64)$ & $60(56-61)$ & $<0.001$ \\
\hline$\alpha$ & $68(63-72)$ & $69(64-72)$ & 0.903 & $72(69-75)$ & $72(69-75)$ & 0.588 \\
\hline AUC & $1183(1112-1350)$ & $1200(1059-1298)$ & 0.245 & $6209(5818-6355)$ & $5915(5593-6104)$ & $<0.001$ \\
\hline
\end{tabular}

ROTEM $^{\circledR}$ parameters: clotting time (CT, s), clot thickness (A5 after 5 min, A10 after 10 min, A15 after 15 min, A30 after 30 min, respectively, $\mathrm{mm}$ ), clot formation time (CFT, s), maximum clot firmness (MCF, mm), alpha ( $\alpha$, angle) and AUC (area under the curve). 
All results of the ROTEM ${ }^{\circledast}$ analysis of sample A were within normal range (Table 3) (15). Transport through the pneumatic tube system with a low velocity of $3 \mathrm{~m} / \mathrm{s}$ (according to local standard "priority" mode, sample B) produce minimal but statistically significant changes in multiple parameters of EXTEM (A10, A15, A30, MCF and AUC, Table 3) and one parameter of INTEM and APTEM (A30, Table 3). Increase in acceleration forces resulted in changes evident in multiple ROTEM $^{\oplus}$ parameters (Tables 3-5, and Supplemental data Tables 1 and 2). The higher the acceleration forces, the more ROTEM ${ }^{\circledast}$ parameters are influenced, including INTEM and APTEM (Tables 4 and 5). FIBTEM is only minimally or not influenced by high acceleration forces (Table 5). Although these differences are statistically significant, all results of $\mathrm{ROTEM}^{\circledR}$ parameters of
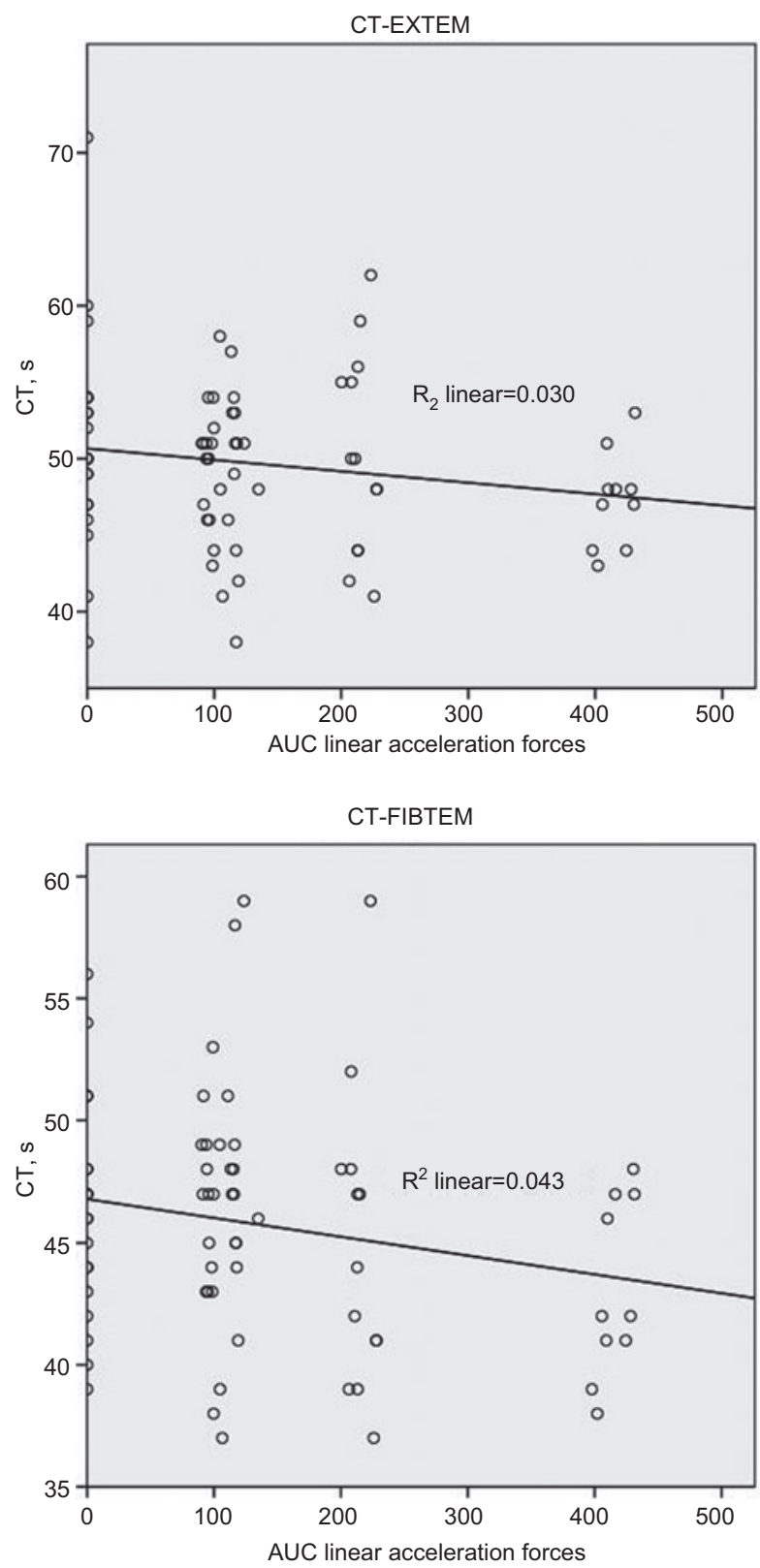

Figure 1 Variation of CT by increasing linear acceleration forces. samples transported via pneumatic tube system are within the normal range. The changes in ROTEM ${ }^{\circledR}$ parameters are more numerous with the increase in linear and rotation acceleration forces (Figures 1-3, and Supplemental data Figure 3). Lysis parameters are influenced only by high acceleration forces (see Supplemental data Table 3).

\section{Discussion}

Originally designed as a point-of-care device, the ROTEManalyser allows a fast evaluation of coagulation function in whole blood. Results of ROTEM ${ }^{\circledR}$ parameters are visualised live on display directly after the start of blood analysis. The

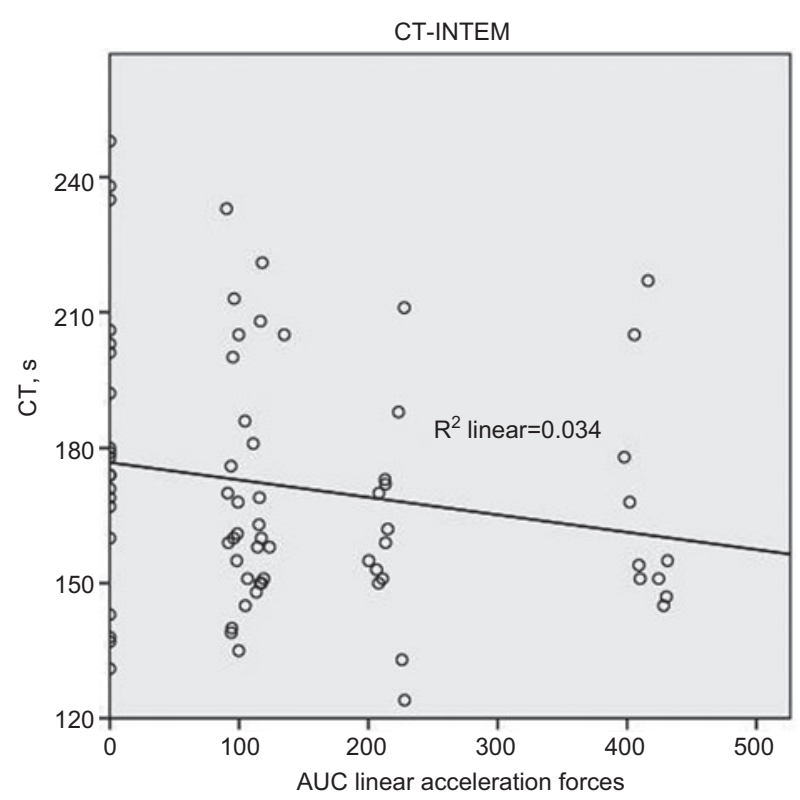

CT-APTEM

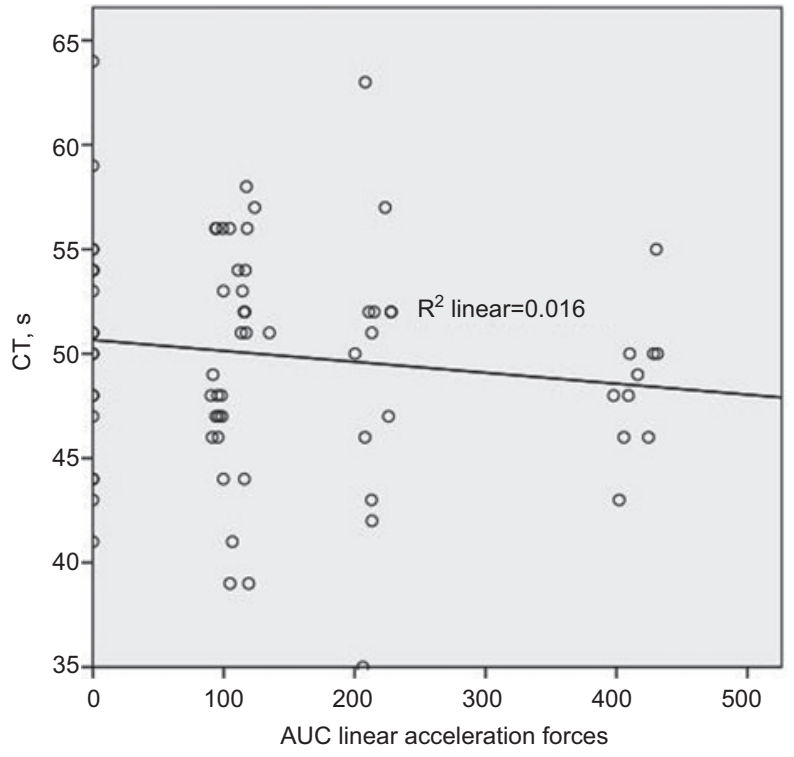



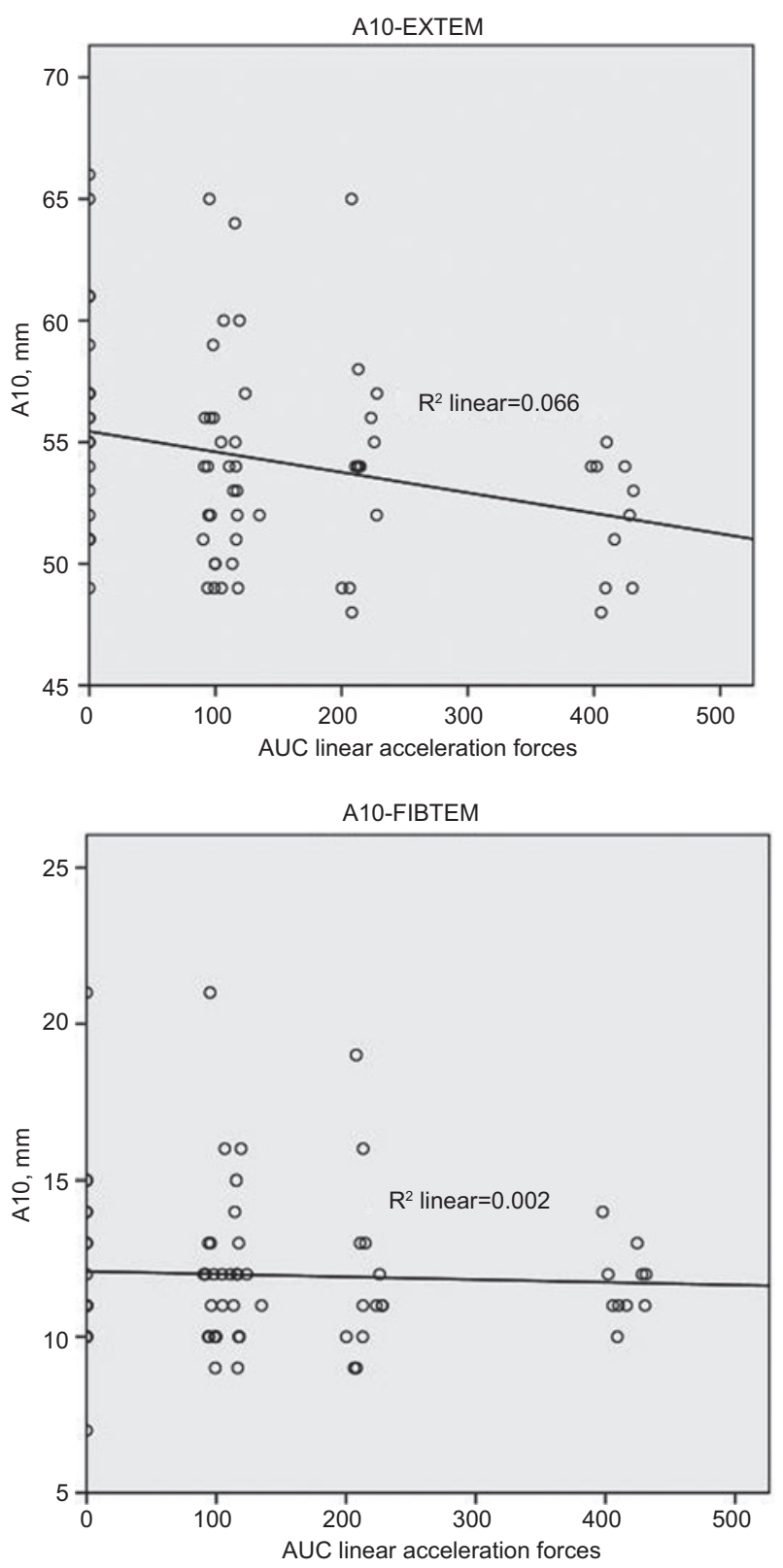

Figure 2 Variation of A10 by increasing linear acceleration forces.

device may be useful, especially in emergency situations with severe bleeding where time is critical, the attending physician needs to obtain information on the type and dimension of a coagulation disorder fast. But often, emergency medical and nursing staff are busy treating the patient, and the performance of laboratory analysis on site may be delayed. Under these circumstances it may be advantageous to perform analysis centrally. Rapid transportation of blood samples to the central laboratory is then a prerequisite. For this purpose pneumatic tube transport systems have been developed and are widely utilised today. To assure quality of analysis, blood coagulation activation by acceleration and deceleration along the trajectory in the pneumatic tube delivery system should be excluded. Comparison of manual and pneumatic delivery of
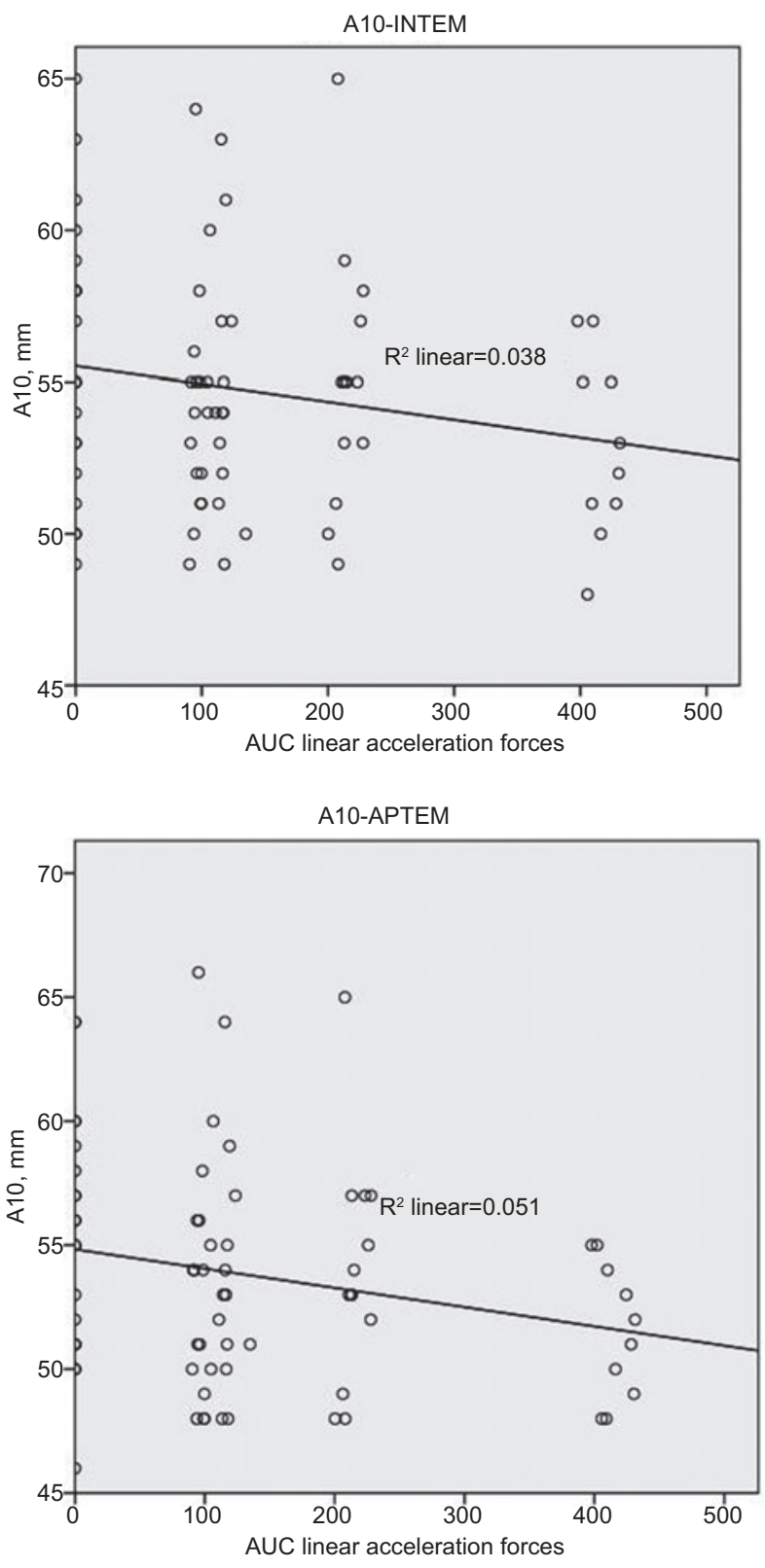

blood samples should be performed to exclude pre-analytical influences $(13,14)$. The first aim of the present study was to investigate the effect of transport on ROTEM $^{\circledR}$ parameters. We show that the mode of transport can influence the results. The second aim of this work was to compare blood sample transport via pneumatic tube vs. manual and we show that the differences, although minimal are significant. At our institution, samples being sent to the central laboratory unit have a slow velocity (of $3 \mathrm{~m} / \mathrm{s}$ ) with a special, direct "priority-transport". Our findings show that although all results were within the normal ranges, the transport through the pneumatic system had influence on the multiple ROTEM ${ }^{\circledR}$ parameters. Finally, to determine the effect of acceleration forces on ROTEM ${ }^{\circledR}$ results we used different speed and distances resulting in 

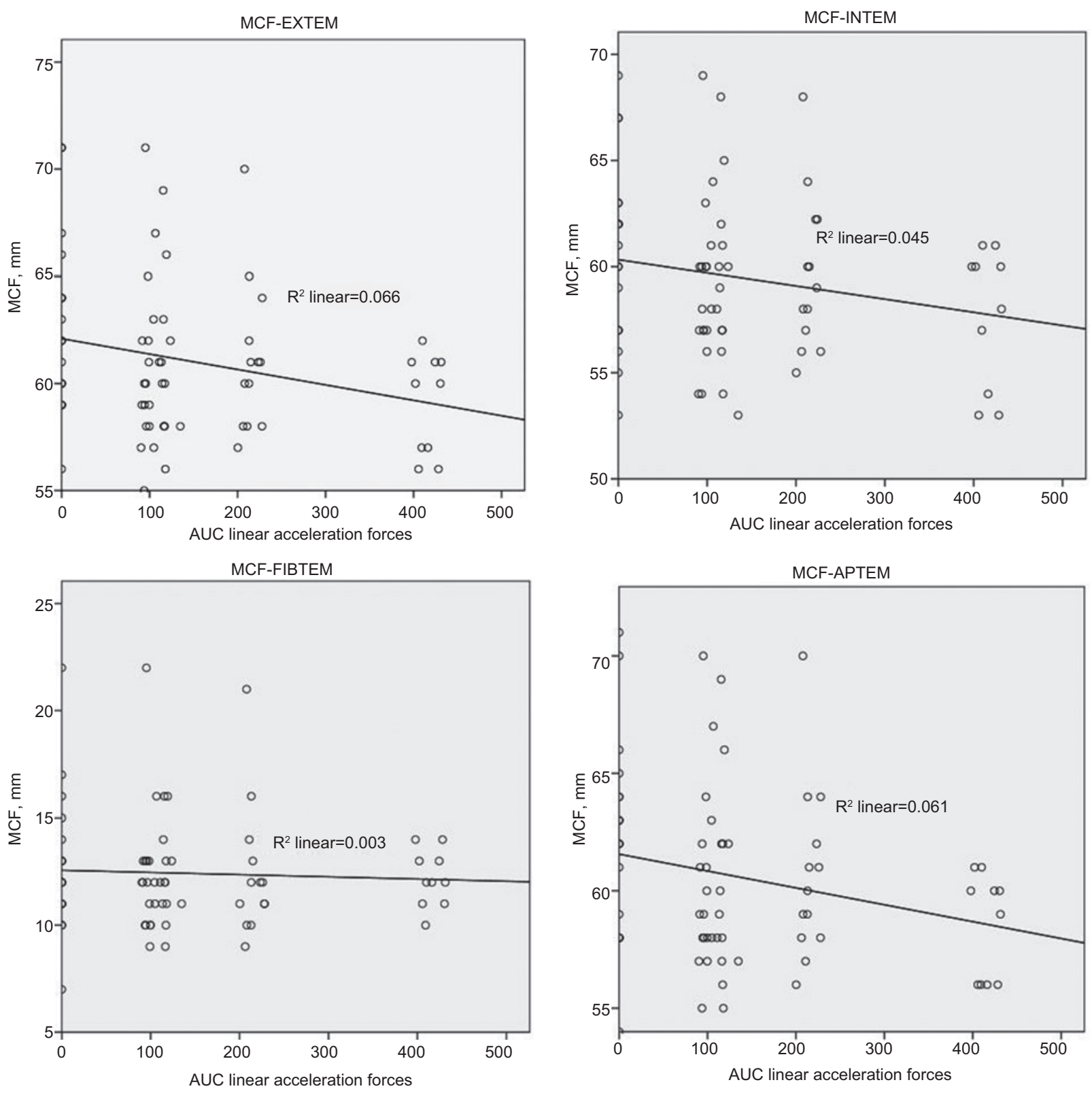

Figure 3 Variation of MCF by increasing linear acceleration forces.

augmentation of acceleration forces. To measure the acceleration forces we used two different accelerometers (16). We show that an increase in acceleration forces resulted in variation of multiple ROTEM $^{\circledR}$ parameters, especially amplitude (A) and maximum clot firmness (MCF). The changes in the ROTEM $^{\circledR}$ parameters are more numerous with the increase in linear and rotation acceleration forces. Interestingly, the FITBEM is influenced only minimally, even with high acceleration forces. The FIBTEM is like the EXTEM but presence of cytochalasin D blocks the contribution of platelets to whole blood coagulation. It is not excluded, that the result observed in this study could be caused by the alteration of thrombocyte function. Of note, the FIBTEM is probably the ROTEM ${ }^{\circledR}$ parameter which mostly affects patients' management.
This study reinforces the thesis, that comparison of manual and local pneumatic delivery should be performed to exclude relevant in vitro blood coagulation activation (14). At each institution an evaluation of the transport system is necessary before samples are routinely transported with a pneumatic tube system. This evaluation is needed at each institution because the transport systems are different (dependent on architectural, length differences, acceleration forces, ...) and every single system should be considered as a unique entity. In the present work all results are within the normal range and differences are probably clinically not relevant. However, this conclusion cannot be extrapolated to patients with bleeding disorders. The comparison should be performed with patient samples as well. An additional weakness of this study is the time difference between 
blood collection, transportation and ROTEM ${ }^{\circledR}$ analysis. This difference was due to the availability of only two ROTEM ${ }^{\circledR}$ devices. However, prior publications show stability of blood samples until $4 \mathrm{~h}$ after sampling $(15,17)$. In addition, our results show a difference already between manual and standard "priority" mode and this difference slightly increases with acceleration and rotation forces. These results are discrepant but not contradictory to our previously published work (14) because the studies are conducted at two different institutions with different tube transport systems and different acceleration forces.

In conclusion, acceleration forces during transport through a pneumatic tube system influence ROTEM $^{\circledR}$ parameters in whole blood samples in healthy volunteers. Prior to the transfer of blood samples via a pneumatic tube system these influences should be tested to exclude clinically relevant preanalytical blood activation in vitro.

\section{Acknowledgments}

We thank Axon Lab for lending ROTEM ${ }^{\circledR}$ delta devices and reagents. Further we thank Jan Kipping, Darwin Lavric, Peter Schumacher (consulting engineers) and Lorenzo Alberio, for their help and advice regarding motion values. Giuseppe Colucci is supported by an unrestricted grant of CSL Behring.

\section{Conflict of interest statement}

Authors' conflict of interest disclosure: The authors stated that there are no conflicts of interest regarding the publication of this article.

Research funding: None declared.

Employment or leadership: None declared.

Honorarium: None declared.

\section{References}

1. Nielsen VG. A comparison of the Thrombelastograph and the ROTEM. Blood Coagul Fibrinolysis 2007;18:247-52.

2. Rugeri L, Levrat A, David JS, Delecroix E, Floccard B, Gros A, et al. Diagnosis of early coagulation abnormalities in trauma patients by rotation thrombelastography. J Thromb Haemost 2007;5:289-95.

3. Fernandes CM, Worster A, Eva K, Hill S, McCallum C. Pneumatic tube delivery system for blood samples reduces turnaround times without affecting sample quality. J Emerg Nurs 2006;32:139-43.

4. Wallin O, Soderberg J, Grankvist K, Jonsson PA, Hultdin J. Preanalytical effects of pneumatic tube transport on routine haematology, coagulation parameters, platelet function and global coagulation. Clin Chem Lab Med 2008;46:1443-9.

5. Koessler J, Kobsar AL, Brunner K, Stolz H, Dossler B, Walter $\mathrm{U}$, et al. The preanalytical influence of two different mechanical transport systems on laboratory analysis. Clin Chem Lab Med 2011;49:1379-82.

6. Pragay DA, Fan P, Brinkley S, Chilcote ME. A computer directed pneumatic tube system: its effects on specimens. Clin Biochem 1980;13:259-61.

7. Astles JR, Lubarsky D, Loun B, SedorFA, Toffaletti JG. Pneumatic transport exacerbates interference of room air contamination in blood gas samples. Arch Pathol Lab Med 1996;120:642-7.

8. Victor Peter J, Patole S, Fleming JJ, Selvakumar R, Graham PL. Agreement between paired blood gas values in samples transported either by a pneumatic system or by human courier. Clin Chem Lab Med 2011;49:1303-9.

9. Collinson PO, John CM, Gaze DC, Ferrigan LF, Cramp DG. Changes in blood gas samples produced by a pneumatic tube system. J Clin Pathol 2002;55:105-7.

10. Dyszkiewicz-Korpanty A, Quinton R, Yassine J, Sarode R. The effect of a pneumatic tube transport system on PFA-100 trade mark closure time and whole blood platelet aggregation. J Thromb Haemost 2004;2:354-6.

11. Hubner U, Bockel-Frohnhofer N, Hummel B, Geisel J. The effect of a pneumatic tube transport system on platelet aggregation using optical aggregometry and the PFA-100. Clin Lab 2010;56:59-64.

12. Bolliger D, Seeberger MD, Tanaka KA, Dell-Kuster S, Gregor $\mathrm{M}$, Zenklusen U, et al. Pre-analytical effects of pneumatic tube transport on impedance platelet aggregometry. Platelets 2009;20:458-65.

13. Plebani M, Zaninotto M. Pneumatic tube delivery systems for patient samples: evidence of quality and quality of evidence. Clin Chem Lab Med 2011;49:1245-6.

14. Colucci G, Giabbani E, Barizzi G, Urwyler N, Alberio L. Laboratory-based $\operatorname{ROTEM}((\mathrm{R}))$ analysis: implementing pneumatic tube transport and real-time graphic transmission. Int J Lab Hematol 2011;33:441-6.

15. Lang T, Bauters A, Braun SL, Potzsch B, von Pape KW, Kolde $\mathrm{HJ}$, et al. Multi-centre investigation on reference ranges for ROTEM thromboelastometry. Blood Coagul Fibrinolysis 2005;16:301-10.

16. Streichert T, Otto B, Schnabel C, Nordholt G, Haddad M, Maric $\mathrm{M}$, et al. Determination of hemolysis thresholds by the use of data loggers in pneumatic tube systems. Clin Chem 2011;57: 1390-7.

17. Zurcher M, Sulzer I, Barizzi G, Lammle B, Alberio L. Stability of coagulation assays performed in plasma from citrated whole blood transported at ambient temperature. Thromb Haemost 2008;99:416-26. 